\title{
Risk Factors for Outlet Obstruction in Patients with Diverting Ileostomy Following Rectal Surgery
}

\author{
Tomoki Abe, Junichi Nishimura, Masayoshi Yasui, Chu Matsuda, Naotsugu Haraguchi, Nozomu Nakai, Hiroshi Wada, \\ Hidenori Takahashi, Takeshi Omori, Hiroshi Miyata and Masayuki Ohue
}

Department of Gastroenterological Surgery, Osaka International Cancer Institute, Osaka, Japan

\begin{abstract}
Objectives: Anastomotic leakage is one of the most severe complications of rectal cancer surgery. A diverting ileostomy was constructed for the purpose of reducing anastomotic failure risk. Outlet obstruction (OO) is one of the complications of diverting stoma that results in a lack of fecal discharge from the stoma. Detailed etiologies and preventive measures for outlet obstruction have not yet been identified.

Methods: We studied 125 patients who underwent rectal resection, anastomosis, and elective ileostomy. We evaluated the incidence of outlet obstruction and looked for any relationship between perioperative factors and outlet obstruction.

Results: Outlet obstruction was detected in 20 cases (16.0\%). Outlet obstruction occurred 9 days after surgery in most cases. Inserting a decompressing tube improved obstructive symptoms in 4 days. Patients were divided into two cohorts according to the occurrence of outlet obstruction. Postoperative hospital stay was longer in the outlet obstruction group (19 vs. 15 days; $p=0.0003)$. A multivariate analysis identified that younger patients, a postoperative thicker rectus abdominis muscle at the stoma passage and high output syndrome were independent risk factors for outlet obstruction.

Conclusions: Younger patients, a postoperative thicker rectus abdominis muscle at stoma passage and high output syndrome were independent risk factors for outlet obstruction.
\end{abstract}

\section{Keywords}

outlet obstruction, rectal cancer, diverting stoma, high output syndrome

J Anus Rectum Colon 2021; 5(3): 254-260

\section{Introduction}

In recent years, the technique of lower rectal cancer surgery for anal preservation has advanced and spread. However, the incidence of anastomotic leakage is reported to be around $10 \%[1,2]$. Anastomotic leakage is one of the most severe postoperative complications and can lead to sepsis and permanent ostomy. Moreover, anastomotic leakage increases the rate of local and distant recurrence[3]. A diverting ileostomy is constructed for the purpose of reducing anastomotic failure risk[4]. By contrast, ileostomy itself is known to be associated with problems such as parastomal hernia, prolapse, skin disorder, high output syndrome (HOS), and outlet obstruction (OO)[5,6]. OO is often found in cases of lack of fecal discharge. According to previous reports, OO occurred in 7.0\%-16.8\% of patients who underwent rectal cancer or ulcerative colitis surgery with diverting ileostomy[7-9]. OO may lead to postponement of oral intake, unplanned stoma closure, and delay of postoperative adjuvant chemotherapy. Some reports have indicated open surgery, torsion of the intestine, and the thickness of the rectus abdominis as risk factors for $\mathrm{OO}[10,11]$. However, de- 
tailed etiologies and preventive measures for $\mathrm{OO}$ have not yet been identified.

In this paper, we report the frequency of $\mathrm{OO}$ and reveal the preoperative and intraoperative risk factors for $\mathrm{OO}$ after lower rectal cancer surgery.

\section{Methods}

\section{Patients}

Cases of all 125 patients who underwent rectal resection, anastomosis, and elective ileostomy for rectal neoplasm between April 2014 and March 2020 at our institution were retrospectively studied.

\section{Method of ileostomy construction}

In all patients for whom an ileostomy was planned, the stoma site was marked before surgery according to the principle of the Cleveland Clinic. An approximately $2 \mathrm{~cm}$ longitudinal incision was made at the stoma site. A $3 \mathrm{~cm}$ longitudinal incision was made at the anterior sheath, the rectus abdominis muscle and the posterior sheath. These steps were performed so that a stoma tunnel was created vertically from the skin to the abdominal cavity. In some cases, a stoma was placed at the umbilicus. In those cases, the stoma was raised from the wound of the port placed at the umbilicus. The ileum was raised approximately $30-40 \mathrm{~cm}$ orally from the Bauhin valve or the anastomotic portion and oriented to the intestine without torsion. The serosa and muscle layer of the intestine were not fixed to the anterior sheath of the rectus abdominis. An incision was made in the raised intestine and with 12 to 16 stitches using 3-0 absorbable suture materials.

\section{Diagnosis of HOS and $\mathrm{OO}$}

Previous studies[7,12,13] defined HOS as a stoma output of $\geq 1,500-2,000 \mathrm{ml} / 24 \mathrm{~h}$. Based on a previous report[12] that HOS with an output $\geq 2,000 \mathrm{ml}$ can cause renal dysfunction, in this study, we defined HOS as an output $\geq 2,000$ $\mathrm{ml} / 24 \mathrm{~h}$.

OO has three ileus signs/symptoms[11]: 1) abdominal distension and vomiting, 2) small intestinal dilation being reduced at the site where the stoma abdominal wall is penetrated, indicated by computed tomography (CT), and 3) the signs/symptoms being reduced by decompression by inserting a tube from the stoma. If there are 1$)+2$ ) or 1$)+3$ ) signs/symptoms, the patient is diagnosed as having OO.

\section{Clinical and biochemical parameter}

We collected clinical data of all patients, including patients' characteristics, laboratory investigations, surgical factors, postoperative factors, and imaging data. Clinical factors were compared between cases with and without OO.

\section{Quantitative assessment of the CT images}

CT scans were obtained before and after surgery. All patients underwent CT scans before the first surgery. We assessed preoperative thickness of subcutaneous fat at the umbilicus and that of the rectus abdominis muscle at the umbilicus. Patients with outlet obstruction underwent CT scans with a slice thickness of 1-5 mm when signs/symptoms appeared. Patients without OO underwent CT scans 3-6 months later at the time of examination for postoperative recurrence. For the ileostomy, we examined the thickness of the rectus abdominis muscle at the level of the stoma passage (Figure 1a) and at the level of the umbilicus (Figure 1 b). We compared these indexes between cases with and without OO.

The images were analyzed using the Aquarius Intuition Server software program version 4.0 (TeraRecon Inc., Tokyo, Japan) by two investigators (TA and JN) who were blinded to the patients' clinical information.

\section{Statistical analysis}

The data are expressed as means \pm standard deviations. Categorical variables were compared using the chi-squared test, and continuous variables were compared using Student's t-test, as appropriate. A multivariate analysis was performed using logistic regression. The optimal cut-off values of the continuous variables were determined using receiver operating characteristic (ROC) curve analysis, if necessary. All analyses were conducted using the JMP 13 software program (SAS Institute, Cary, NC, USA). Any variable deemed significant $(p<0.05)$ in the univariate analysis was a candidate for multivariate analysis. Statistical significance was defined as a $p$-value $<0.05$.

\section{Consent}

Written consent was obtained from all patients and relevant persons (such as the parents or legal guardians) to publish the information, including photographs.

\section{Ethical guidelines}

This study was approved by the Human Ethics Review Committee of the Osaka International Cancer Institute (no. 18033).

\section{Results}

This study included 125 patients, and the patient characteristics are listed in Table 1. One hundred twenty patients (96\%) underwent surgery for rectal cancer, and the others were for gastrointestinal stromal tumor or neuroendocrine tumor. Robot-assisted surgery was performed in 24 cases; laparoscopic surgery, in 79 cases; and open surgery, in 22 cases. Rectal resection was performed in 123 cases, and to- 


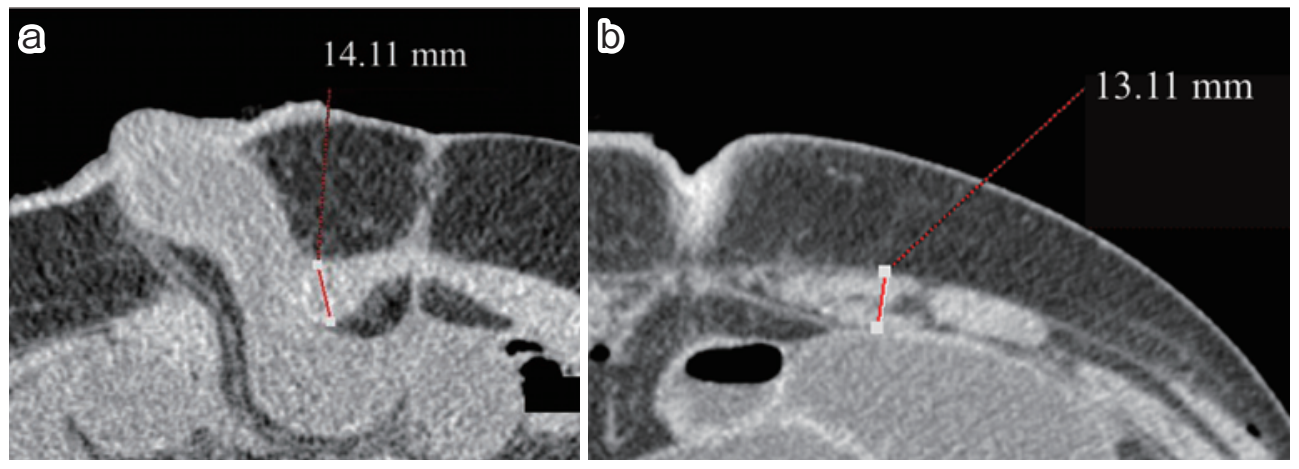

Figure 1.

a. CT analysis of the thickness of the rectus abdominis at the level of the stoma passage.

By using the CT image at the level of the stoma passage, the thickest part of the rectus abdominis muscle was measured perpendicular to the straight line connecting both ends of the rectus abdominis muscle. The thickness of the rectus abdominis at the level of the stoma passage in this case was $14.11 \mathrm{~mm}$.

b. CT analysis of the thickness of the rectus abdominis at the level of the umbilicus.

By using the CT image at the level of the umbilicus, the thickest part of the rectus abdominis muscle was measured perpendicular to the straight line connecting both ends of the rectus abdominis muscle. The thickness of the rectus abdominis at the level of the umbilicus in this case was $13.11 \mathrm{~mm}$.

Table 1. Clinicopathological Characteristics of the 125 Patients.

\begin{tabular}{lc}
\hline \multicolumn{1}{c}{ Patient factors } & Median (range) or $\mathrm{n}$ \\
\hline Age (years) & $60(32-84)$ \\
Gender (male/female) & $78 / 47$ \\
BMI $\left(\mathrm{kg} / \mathrm{m}^{2}\right)$ & $22.2(15.6-35.0)$ \\
Prior abdominal surgery (positive/negative) & $29 / 96$ \\
Preoperative thickness of subcutaneous fat at the umbilicus & $17.3(2.3-42.3)$ \\
Preoperative thickness of rectus abdominis muscle at the umbilicus & $9.2(3.5-15.5)$ \\
Postoperative thickness of subcutaneous fat at the umbilicus $(\mathrm{mm})$ & $24.1(7.9-49.5)$ \\
Postoperative thickness of the rectus abdominis muscle at the umbilicus $(\mathrm{mm})$ & $10.3(5.5-17.1)$ \\
Thickness of the rectus abdominis muscle at the stoma passage $(\mathrm{mm})$ & $10.4(6.2-19.0)$ \\
DM (positive/negative) & $11 / 114$ \\
Clinical Stage (I/II/III/IV/others) & $60 / 28 / 28 / 4 / 5$
\end{tabular}

\begin{tabular}{lc}
\hline \multicolumn{2}{c}{ Surgical factors } \\
\hline Operative time (min) & $414(162-786)$ \\
Estimated blood loss (ml) & $50(0-2290)$ \\
Approach (robot/laparoscopy/laparotomy) & $24 / 79 / 22$ \\
Procedure (LAR/sLAR/ISR/total proctocolectomy) & $22 / 62 / 39 / 2$ \\
LLND (yes/no) & $39 / 86$ \\
Stomal site (lower right/upper right/lower left/umbilicus) & $115 / 6 / 2 / 2$ \\
Direction of oral ileum (cranial/caudal) & $109 / 12$ \\
\hline \multicolumn{2}{c}{ Postoperative factors } \\
Stoma outlet obstruction (yes/no) & $20(16.0 \%) / 104(84.0 \%)$ \\
Surgical site infection (yes/no) & $3 / 122$ \\
HOS (positive/negative) & $18 / 107$ \\
Postoperative hospital stay (days) & $15(9-51)$ \\
\hline
\end{tabular}

BMI: body mass index, DM: diabetes mellitus, LAR: lower anterior resection, sLAR: super lower anterior resection, ISR: intersphincteric resection, LLND: lateral lymph node dissection, HOS: high output syndrome 
Table 2. Analysis for Patients with Outlet Obstruction Group.

\begin{tabular}{lc}
\hline & Median (range) or $\mathrm{n}$ \\
\hline Days of incidence of outlet obstruction (days) & $7(0-29)$ \\
Intervention (drainage/none) & $15 / 5$ \\
Days of recovery from outlet obstruction (days) & $4(2-10)$ \\
\hline
\end{tabular}

Table 3. Univariate Analysis for the Incidence of Outlet Obstruction.

\begin{tabular}{|c|c|c|c|}
\hline Patient factors & $\begin{array}{l}\text { OO group }(\mathrm{n}=20) \\
\text { Median (range) or } \mathrm{n}\end{array}$ & $\begin{array}{l}\text { nOO group }(\mathrm{n}=105) \\
\text { Median (range) or } \mathrm{n}\end{array}$ & $\begin{array}{c}\text { Univariate analysis } \\
(p \text {-value })\end{array}$ \\
\hline Age (years) & $55.5(36-74)$ & $62.0(32-84)$ & 0.040 \\
\hline Gender (male/female) & $12 / 8$ & $66 / 39$ & 0.81 \\
\hline $\operatorname{BMI}\left(\mathrm{kg} / \mathrm{m}^{2}\right)$ & $21.7(17.6-26.8)$ & $22.3(15.6-35.0)$ & 0.42 \\
\hline Prior abdominal surgery (positive/negative) & $3 / 17$ & $26 / 79$ & 0.32 \\
\hline Preoperative thickness of subcutaneous fat at the umbilicus & $20.1(11.4-29.5)$ & $15.8(2.3-42.3)$ & 0.12 \\
\hline Preoperative thickness of rectus abdominis muscle at the umbilicus & $10.1(5.9-15.5)$ & $9.1(3.5-15.2)$ & 0.17 \\
\hline Postoperative thickness of subcutaneous fat at the umbilicus (mm) & $23.2(12.4-38.7)$ & $24.2(7.9-49.5)$ & 0.82 \\
\hline Postoperative thickness of rectus abdominis muscle at the umbilicus ( $\mathrm{mm}$ ) & $11.5(6.7-15.1)$ & $10.3(5.5-17.1)$ & 0.14 \\
\hline Thickness of the rectus abdominis muscle at the stoma passage (mm) & $12.1(7.1-15.7)$ & $10.2(6.2-19.0)$ & 0.0024 \\
\hline DM (positive/negative) & $0 / 20$ & $6 / 99$ & 0.14 \\
\hline Clinical Stage (I/II/III/IV/others) & $10 / 4 / 4 / 1 / 1$ & $50 / 24 / 24 / 3 / 4$ & 0.98 \\
\hline \multicolumn{4}{|l|}{ Surgical factors } \\
\hline Operative time (min) & $427(249-579)$ & $409(162-786)$ & 0.56 \\
\hline Estimated blood loss (ml) & $20(0-1,355)$ & $55(0-2,290)$ & 0.90 \\
\hline Approach (robot/laparoscopy/laparotomy) & $5 / 12 / 3$ & $19 / 67 / 19$ & 0.77 \\
\hline Procedure (LAR/sLAR/ISR/total proctocolectomy) & $5 / 10 / 4 / 1$ & $17 / 51 / 36 / 1$ & 0.092 \\
\hline LLND (yes/no) & $5 / 15$ & $43 / 62$ & 0.39 \\
\hline Stomal site (lower right/upper right/lower left/umbilicus) & $17 / 1 / 1 / 1$ & $98 / 5 / 1 / 1$ & 0.49 \\
\hline Direction of oral ileum (cranial/caudal) & $19 / 1$ & $90 / 11$ & 0.39 \\
\hline \multicolumn{4}{|l|}{ Postoperative factors } \\
\hline Surgical site infection (yes/no) & $1 / 19$ & $2 / 103$ & 0.46 \\
\hline HOS (positive/negative) & $7 / 13$ & $11 / 94$ & 0.0096 \\
\hline Postoperative hospital stay (days) & $19(14-45)$ & $15(9-51)$ & 0.0003 \\
\hline
\end{tabular}

BMI: body mass index, DM: diabetes mellitus, LAR: lower anterior resection, sLAR: super lower anterior resection, ISR: intersphincteric resection, LLND: lateral lymph node dissection, HOS: high output syndrome, OO: outlet obstruction

tal proctocolectomy was performed in 2 cases. In the majority of cases, the stoma was constructed in the lower right $(92 \%)$. Twenty cases $(16.0 \%)$ had a clinical diagnosis of OO. HOS occurred in 18 patients $(14.4 \%)$.

Table 2 presents the analysis for patients with OO. The median time to $\mathrm{OO}$ occurrence was 7 days (range: 0-29) after surgery. In most cases $(90.5 \%)$, OO occurred within 9 days after surgery (Figure 2). Drainage was performed in 15 patients $(75.0 \%)$ of cases after the onset of OO. In the case of $\mathrm{OO}$, inserting a decompression tube to the oral side of the intestine improved the obstructive symptoms. Median improvement in days from $\mathrm{OO}$ was 4 days (range: 2-10 days). All 15 cases inserting the decompression tube recovered in 3 days (range: 0-10) (data not presented).

Table 3 summarizes the clinical characteristics of the two groups; the cohort of the patients with $\mathrm{OO}$ was named the "OO group," and the cohort of the patients without $\mathrm{OO}$ was named the "nOO group." The comparison of the two groups detected significant differences in three parameters: age (scores in the $\mathrm{OO}$ and nOO groups were 55.5 and 62.0, respectively, $p=0.040$ ); postoperative thickness of the rectus abdominis muscle at the stoma passage (scores in the $\mathrm{OO}$ and $\mathrm{nOO}$ groups were 12.1 and 10.2, respectively, $p=$ 0.0024); and incidence of HOS (the proportions of patients with $\mathrm{HOS}$ in the $\mathrm{OO}$ and $\mathrm{nOO}$ groups were $35.0 \%$ and $10.5 \%$, respectively, $p=0.0096)$. In the OO group, all patients received CT scans before closing ileostomy. In the nOO group, CT scans before closing ileostomy were made in 92 patients $(87.6 \%)$. In the OO group, postoperative hospital stay was significantly extended (scores in the $\mathrm{OO}$ and 
Table 4. Multivariate Analysis for the Incidence of Outlet Obstruction.

\begin{tabular}{|c|c|c|c|}
\hline & $P$-value & Odds ratio & $95 \% \mathrm{CI}$ \\
\hline Age $(<60 / \geqq 60)$ (years) & 0.021 & 4.72 & $1.27-17.53$ \\
\hline $\begin{array}{l}\text { Postoperative thickness of the rectus abdominis muscle } \\
\text { at the stoma passage }(\geqq 10.4 /<10.4)(\mathrm{mm})\end{array}$ & 0.0067 & 4.93 & $1.56-15.64$ \\
\hline HOS (positive/negative) & 0.0058 & 6.84 & $1.75-26.77$ \\
\hline
\end{tabular}

HOS: high output syndrome

$$
\text { (cases) } 5
$$

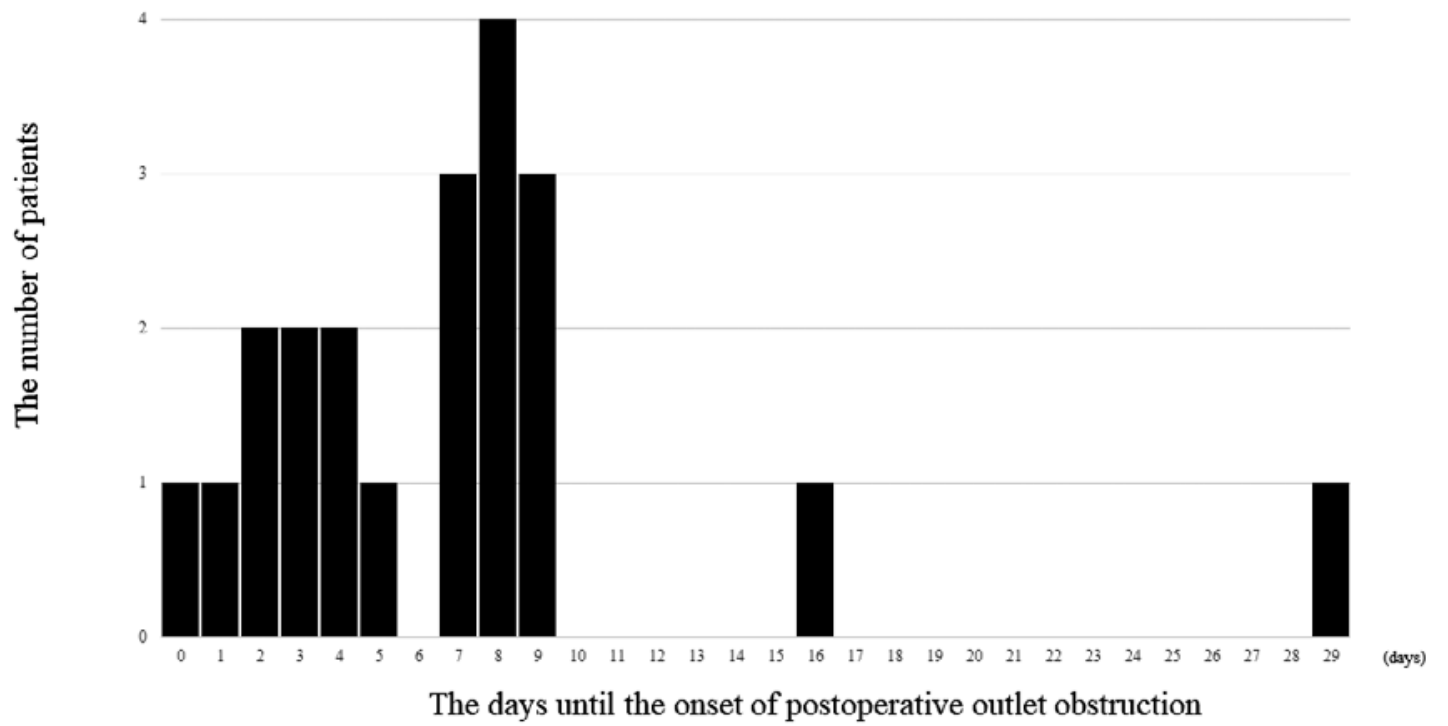

Figure 2. Days from onset of outlet obstruction to disappearance of symptoms.

In most cases $(\mathrm{n}=19 ; 90.4 \%)$, symptoms improved within 9 days.

nOO groups were 19 and 15 , respectively, $p=0.0003$ ). Surgical approach, operative time, direction of oral ileum (cranial or caudal) and the incidence of surgical site infection were not affected by the incidence of OO.

The preoperative rectus abdominis muscle at the umbilicus (9.7 vs. 8.9; $\mathrm{p}=0.02)$, the postoperative rectus abdominis muscle at the umbilicus $(11.5$ vs. $9.7 ; \mathrm{p}<0.0001)$ and the rectus abdominis muscle at stoma passage (11.3 vs. 9.9; $\mathrm{p}=0.003$ ) were significantly thicker in young patients (under 60 years old). The other factors (e.g., thickness of subcutaneous fat, body mass index and high output syndrome) were not associated with age.

The multivariate analysis was performed with the three significant factors that were statistically significant in the univariate analyses (Table 4). Cut-off values were set using the median values; age. All three parameters were found to be independent risk factors significantly associated with the incidence of $\mathrm{OO}$.

\section{Discussion}

The purpose of constructing a diverting stoma is mostly to prevent postoperative anastomotic leakage in rectal cancer or to change the stool passage in the treatment after postoperative anastomotic failure. The effectiveness of constructing a diverting stoma has been proven by a meta-analysis[14]. In our department, all ileostomy constructions were intended for the prevention of anastomotic leakage.

It is considered that the pressure of the rectus abdominis muscle prevents stool excretion from the stoma. Kanazawa et al.[15] reported that $\mathrm{OO}$ is more common in patients with thick rectus abdominis muscles $(\geq 10 \mathrm{~mm})$. By contrast, Fujii et al.[11] reported that the higher thickness of the rectus abdominis muscles at the stoma-penetrating site was unfavorably related to OO. Similarly, in this study, OO was significantly higher in patients with thin rectus abdominis muscles "in general" but with thick rectus abdominis muscles "at stoma passage." As the thickness of the muscle increases in the center of the rectus abdominis, it is better to create a 
stoma on the outer side of the rectus abdominis. Especially, young patients need to be careful that the rectus abdominis muscle is thick. When the rectus abdominis muscle is thick, it is expected that the intestinal pressure will be overwhelmed by the pressure of the rectus abdominis muscle, hindering feces excretion. Ileostomy is reported to have more OO than colostomy, and it is speculated that the intraluminal pressure of intestine is lower than that of the colon[16,17].

Tang et al. reported that the amount of stoma output peaked on the fourth day after surgery and decreased by the ninth day. In our study, in almost all cases, OO occurred within 9 days after creation of the stoma. It is speculated that $\mathrm{OO}$ is caused by not only the narrowing of the intestinal tract through the abdominal wall but also by relative narrowing due to HOS. HOS was significantly higher in the laparotomy group and may be a predictor of postoperative HOS. Takeda et al.[13] also reported that HOS was significantly associated with diabetes mellitus and total proctocolectomy. Hara et al.[18] reported that infection in organ/ space sites is associated with HOS. However, in this study, these factors were not associated with HOS. Further investigation is necessary to identify the causes of HOS.

In the case of $\mathrm{OO}$, inserting a decompression tube to the oral side of the intestine can improve the obstructive symptoms. In this study, immediate improvement was observed in the decompression tube insertion cases. From the above, it was speculated that the cause of $\mathrm{OO}$ was the narrowing (including relative narrowing) of the stoma site tunnel. Once OO occurs, the hospital stay will be extended, and in some cases, $\mathrm{OO}$ is not improved by inserting a decompression tube, in which case the surgeon may be forced to close the stoma.

As a measure against OO, Uchino et al.[19] reported that it is more effective to perform fascial incision as a longitudinal incision than to make a cruciate incision and to orient the oral side of the ascending intestinal tract in the 3 o'clock direction to reduce twisting of the mesentery.

The limitation of this study was that it was a singlecenter, retrospective, observational study. A larger, longerterm, prospective study is required to identify more clearly the factors that contribute to OO.

Diverting ileostomy was constructed to reduce the risk of anastomotic insufficiency, but $\mathrm{OO}$ occurred in $17 \%$ of patients. OO was associated with certain factors (age, thickness of the rectus abdominis at stoma passage and HOS). It is necessary to further identify clearly factors that contribute to $\mathrm{OO}$ so that different surgical techniques can be employed to prevent $\mathrm{OO}$.

In conclusion, younger patients, thicker rectus abdominis muscle at stoma passage and high output syndrome were independent risk factors for outlet obstruction.
Conflicts of Interest

There are no conflicts of interest.

\section{Author Contributions}

Tomoki Abe, MD and Junichi Nishimura, MD, PhD contributed to the conception, design, the acquisition, analysis interpretation of data for the work.

Masayoshi Yasui, MD, $\mathrm{PhD}$; Chu Matsuda, $\mathrm{MD}, \mathrm{PhD}$; Naotsugu Haraguchi, MD, PhD; Nozomu Nakai, MD; Hiroshi Wada, MD, PhD; Hidenori Takahashi, MD, PhD; Takeshi Omori, MD, PhD; Hiroshi Miyata, MD, PhD; and Masayuki Ohue, $\mathrm{MD}, \mathrm{PhD}$ contributed to the acquisition and analysis of data.

Approval by Institutional Review Board (IRB)

18033

The Human Ethics Review Committee of the Osaka International Cancer Institute

\section{References}

1. Paun BC, Cassie S, MacLean AR, et al. Postoperative complications following surgery for rectal cancer. Ann Surg. 2010 May; 251(5): 807-18.

2. Tsai YY, Chen WT. Management of anastomotic leakage after rectal surgery: a review article. J Gastrointest Oncol. 2019 Dec; 10 (6): 1229-37.

3. Koedam TWA, Bootsma BT, Deijen CL, et al. Oncological outcomes after anastomotic leakage after surgery for colon or rectal cancer: increased risk of local recurrence. Ann Surg. 2020 Mar 27; published ahead of print.

4. Mrak K, Uranitsch S, Pedross F, et al. Diverting ileostomy versus no diversion after low anterior resection for rectal cancer: a prospective, randomized, multicenter trial. Surgery. 2016 Apr; 159(4): 1129-39.

5. Mizushima T, Kameyama H, Watanabe K, et al. Risk factors for small bowel obstruction following total proctocolectomy and ileal pouch anal anastomosis with diverting loop-ileostomy for ulcerative colitis. Ann Gastroenterol Surg. 2017 Jun; 1(2): 122-8.

6. Shabbir J, Britton DC. Stoma complications: a literature overview. Colorectal Dis. 2010 Oct; 12(10): 958-64.

7. Tamura K, Matsuda K, Yokoyama S, et al. Defunctioning loop ileostomy for rectal anastomoses: predictors of stoma outlet obstruction. Int J Colorectal Dis. 2019 Jun; 34(6): 1141-5.

8. Okada S, Hata K, Emoto S, et al. Elevated risk of stoma outlet obstruction following colorectal surgery in patients undergoing ileal pouch-anal anastomosis: a retrospective cohort study. Surg Today. 2018 Dec; 48(12): 1060-7.

9. Ohira G, Miyauchi $H$, Hayano $K$, et al. Incidence and risk factors for outlet obstruction after construction of ileostomy. J Anus Rectum Colon. 2018; 2(1): 25-30.

10. Eto K, Kosuge M, Ohkuma M, et al. Defunctioning ileostomy is a key risk factor for small bowel obstruction after colorectal cancer resection. Anticancer Res. 2018 Mar; 38(3): 1789-95.

11. Fujii T, Morita H, Sutoh $\mathrm{T}$, et al. Outlet obstruction of temporary loop diverting ileostomy. Hepatogastroenterology. 2015 May; 62 (139): 602-5.

12. Cottam J, Richards $K$, Hasted A, et al. Results of a nationwide 
prospective audit of stoma complications within 3 weeks of surgery. Colorectal Dis. 2007 Nov; 9(9): 834-8.

13. Takeda M, Takahashi H, Haraguchi N, et al. Factors predictive of high-output ileostomy: a retrospective single-center comparative study. Surg Today. 2019 Jun; 49(6): 482-7.

14. Hüser N, Michalski CW, Erkan M, et al. Systematic review and meta-analysis of the role of defunctioning stoma in low rectal cancer surgery. Ann Surg. 2008 Jul; 248(1): 52-60.

15. Kanazawa A, Shiozawa M, Inagaki D, et al. A study on postoperative ileus in patients with ileostomy as a diverting stoma after low anterior resection. Nippon Daicho Komonbyo Gakkai Zasshi. 2009; 62(8): 497-501.

16. Fink S. The intraluminal pressures in the intact human intestine. Gastroenterology. 1959 May; 36(5): 661-71.

17. Chen JH, Yu Y, Yang Z, et al. Intraluminal pressure patterns in the human colon were assessed by high-resolution manometry. Sci Rep. 2017 Feb; 7: 41436.

18. Hara Y, Miura T, Sakamoto Y, et al. Organ/space infection is a common cause of high output stoma and outlet obstruction in diverting ileostomy. BMC Surg. 2020; 20(1): 83.

19. Uchino M, Ikeuchi $H$, Bando T, et al. Efficacy of a cross incision at the rectal muscle fascia and ileostomy rotation for prevention of outlet obstruction at the ostomy site in restorative proctocolectomy for ulcerative colitis. Nippon Daicho Komonbyo Gakkai Zasshi. 2011; 64: 3-77.

Journal of the Anus, Rectum and Colon is an Open Access journal distributed under the Creative Commons Attribution-NonCommercial-NoDerivatives 4.0 International License. To view the details of this license, please visit (https://creativ ecommons.org/licenses/by-nc-nd/4.0/). 